\title{
Why Do Older Korean Adults Respond Differently to Activities of Daily Living and Instrumental Activities of Daily Living? A Differential Item Functioning Analysis
}

\author{
Soong-nang Jang ${ }^{1,2}$, Ichiro Kawachi ${ }^{2}$ \\ ${ }^{1}$ Red Cross College of Nursing, Chung-Ang University, Seoul, Korea \\ ${ }^{2}$ Department of Social and Behavioral Sciences, Harvard T.H. Chan School of Public Health, Boston, MA, USA
}

\section{Corresponding Author:}

Soong-nang Jang, $\mathrm{RN}, \mathrm{PhD}$

Red Cross College of Nursing, Chung-

Ang University, 84 Heukseok-ro

Dongjak-gu Seoul 06974, Korea

E-mail: sjang@cau.ac.kr

ORCID:

https://orcid.org/0000-0003-2621-945X

Received: December 2, 2019

Revised: December 17, 2019

Accepted: December 19, 2019
Objectives: Culture-based gender norms regarding who performs daily activities can bias the assessment of instrumental activities of daily living (IADL). This study evaluated item-response biases in the activities of daily living (ADL) and IADL among community-living Korean older adults. Methods: The subjects included older Korean participants of the Korean Longitudinal Study of Ageing baseline survey $(n=4,164)$. We performed differential item functioning (DIF) analysis of these data using the Mantel-Haenszel method. Results: We observed different reported levels of disability for eight IADL items among gender, age, and educational level subgroups. After matching for overall functional disability, men were more likely to report limitations in performing various household activities, compared to cognitive activities in women. Conclusions: Cross-national comparisons of $A D L$ and IADL disabilities need to consider item response bias stemming from culture-based gender norms regarding who performs different household activities.

Key Words: Activities of daily living, Gender, Item response theory

\section{INTRODUCTION}

The activities of daily living (ADL) and instrumental activities of daily living (IADL) scales represent fundamental measurements of functional status and are important tools not only for planning and predicting the need for long-term care but also for assessing the effectiveness of various policies and services for older populations. ${ }^{1)}$ Several studies have confirmed the accuracy, validity, and reliability of these functional scales. However, their accuracy as a measure of functioning may be limited by latent biases stemming from cultural differences in who performs different activities.

Gender differences in the ADL and IADL tasks have long been postulated. When asked about items related to performing light housework, doing laundry, and preparing meals, men often report that they do not perform these functions due to gender roles. Lawton $^{2)}$ recognized traditional gender roles as possible confounders of accurate functional assessment in the development of the IADL scale and consequently excluded cooking and housekeeping when assessing functional disability in men. However, LaPlante ${ }^{3)}$ suggested that the successful performance of home management chores, such as cooking and cleaning, is necessary to maintain a reasonable standard of living. In another study investigating gender bias in the reported impairment of daily activities in cancer patients, approximately half of the help received by married men was attributable to gender role responsibilities (tasks seen as women's work) rather than functional disability. Role-related help with household tasks was less prevalent among married women. ${ }^{4)}$

The perceived gender roles of older adults may further differ between countries and cultures. In East Asian countries such as Korea, where gender segregation has traditionally been more pronounced than in other countries, gender-specific patterns of item response bias for $\mathrm{ADL} / \mathrm{LADL}$ questionnaires may be particularly 
salient. Moreover, because of gender- and age-based differences in educational attainment, gender may have significant latent effects on the $\mathrm{ADL} / \mathrm{IADL}$ item responses of older generations. Based on studies performed for the development of the Korean versions of $\mathrm{ADL}$ and $\mathrm{IADL},{ }^{5-8)}$ Won et al. ${ }^{6}$ observed that older Korean men reported being unable to do laundry, prepare meals, and perform household chores because they had not previously performed these activities. Likewise, older women were likely to have difficulties managing money, using the telephone, and using transportation because they lacked prior experience with such tasks.

Women in Korea have only recently attained any degree of economic power. ${ }^{9)}$ Social activities such as managing money, banking, using the subway, and driving were previously considered specific male tasks rather than basic functional activities. With dramatically improved and modernized education after World War II and the Korean War, older women of more recent generations and baby boomers may be less subject to gender bias in ADL/LADL responses. However, although they are physically capable of doing these things, many older Korean women are still dependent on their husbands, children, and other family members for tasks such as using cell phones, taking public transport, and using a bank. ${ }^{9}$ Thus, women are likely to report needing help with 'cognitive activities' while being independent in their ability to perform household activities, while older men show an opposite tendency.

Item response theory (IRT) is used in comparison tests and similar instruments to measure unobservable respondent characteristics. IRT models are used extensively in the study of health outcomes and the development of item banks and computerized adaptive testing. ${ }^{10)}$ Eight previous IRT-based studies have revealed multidimensionality, ${ }^{11-13)}$ comparability, ${ }^{14)}$ and group differences ${ }^{11,15)}$ in ADL/LADL responses. Cabrero-Garcia et al. ${ }^{11)}$ suggested a modified aggregated ADL/LADL measure that would account for gender bias in determining the severity of impairment. Items on the IADL list can be divided into household, outdoor, and cognitive activities; based on traditional gender roles, gender bias is particularly strong in household activities. However, unidimensional analyses of $\mathrm{ADL}$ appear to be sufficient in women, while in men, $\mathrm{ADL}$ is bidimensional and divided into self-care and mobility, ${ }^{4,11}$

To more accurately measure and monitor functional status, the use and interpretation of $\mathrm{ADL} / \mathrm{IADL}$ measurements should consider the characteristics of tasks and cultural backgrounds of the respondents. Therefore, we investigated the gender-, educational level-, and age-specific IRTs of ADL/IADL items in older Korean adults and the latent bias in the measurement tool when determining functional status.

\section{MATERIALS AND METHODS}

\section{Data and Sample}

The analyses were based on the baseline wave of the Korean Longitudinal Study of Ageing (KLoSA), a population-based survey of a nationally representative sample of older adults conducted from July to September 2006. All participants aged 65 years or older were included if they responded to ADL/IADL items and reported their educational level. A total of 4,164 subjects were included in the final analyses. The study design and protocol were reviewed and approved by the Chung-Aung University Institutional Review Board (No. 1041078-201708-HRSB-163-01).

\section{Functional Disability}

Functional disability was measured using the 7-item Korean ADL scale and the 10-item Korean IADL scale. ${ }^{8)}$ The ADL scale includes items on getting dressed, washing one's face and hands, bathing, eating meals, leaving a room, using the toilet, and urinating and/or defecating; the IADL scale includes items related to personal grooming, going out for short walks, using transportation, making/receiving phone calls, managing money, doing household chores, preparing meals and cooking, shopping, taking medications, and doing laundry. If the respondents were partly or totally dependent for a given activity, they were categorized as dependent (dependent $=1$, independent $=0$ ). The sum of their $\mathrm{ADL}$ and IADL deficit scores were representative of their functional status. Some controversy remains whether ADL and IADL are unidimensional or multidimensional measures. ${ }^{16)}$ As proposed previously, ${ }^{17,18)}$ we divided ADLs and IADLs into two and three dimensions, respectively. The ADL tasks that included changing clothes, washing, shower, eating, using the toilet, and defecation and urination were categorized as 'self-care'. Items related to getting up and leaving the room were conceptualized as 'mobility'. We considered IADL tasks such as grooming and going out to rely on physical health or strength and, therefore, classified them as 'physical IADLs. Likewise, we considered tasks involving using transportation, managing money, using the telephone, and taking medication to require complex cognitive resources, and, thus, classified them as 'cognitive IADLs'. We categorized other tasks, such as performing light housework, cooking, doing laundry, and shopping, as 'household activities.'.1719)

\section{Statistical Analyses}

Gender, age, and educational level were exogenous variables. Age and education level were dichotomized ( $<75$ vs. $\geq 75$ years and any vs. no education). The total combined ADL/IADL scores were log-transformed to correct for skewness before being used in 
the analyses. We used Mantel-Haenszel (MH) adjustment methods. The MH odds ratio (OR) conditioned on the total scale score is test a commonly used for differential item functioning (DIF) analysis in which both response items and exogenous variables are binary. ${ }^{16,20,21)}$ According to IRT, the responses to a survey item are expected to be the same across the level of the exogenous variables once conditioned on the latent variable. An independent association between any item and any exogenous variable (i.e., if the total scale-adjusted OR is not equal to 1.0) provides evidence that the response to that item is biased with respect to the exogenous variable.

DIF is used when the items intended to measure a latent trait are unfair, favoring one group of individuals over another. DIF is investigated in conjunction with fitting IRT models. DIF includes an assessment that the item behaves differently across respondents with the same value of the latent trait. An item 'functions differently' across individuals with the same latent trait level if these individuals have different probabilities of selecting a given response. ${ }^{22}$

We explored the odds assumption for $\mathrm{p}$-values from the odds score test $<0.05$. As the $\mathrm{p}$-value confounds sample size with effect size, we also followed a previous study on IRT $^{20)}$ by retaining all items with relatively large practically meaningful biases, which we defined as an OR $>2.0$ or $<0.5$. An OR of 2.0 means that those in the test group were twice as likely to provide a higher response (i.e., to report disability) to an individual item than those in the control group after matching for overall functional disability score. To test for item discrimination bias, we included an interaction term between the exogenous variable and the total $\mathrm{ADL} / \mathrm{IADL}$ score for those items with practically meaningful item difficulty bias. We tested for differential factor functioning or factor bias by associating the factor score with each exogenous variable, while conditioning the overall $\mathrm{ADL}$ and IADL scores. The ADL and IADL items were grouped into 5 categories (self-care, mobility, physical activities, cognitive activities, and household activities) based on the results of previous studies. Finally, we calculated the disability rates using the full versions of ADL and IADL as well as shortened revised versions of the ADL and IADL from which we removed items that functioned significantly differently from the exogenous variables (gender, age, and educational level). We calculated the disability rates as the percentage of older adults categorized as dependent in one or more $\mathrm{ADL} / \mathrm{IADL}$ items.

\section{RESULTS}

The mean participant age was $72.9 \pm 6.3$ years. About $35.5 \%$ were aged 75 years or older, $41.8 \%$ were men, and $44.5 \%$ had completed any level of formal education. All item responses were skewed towards the 'do not need help' category. The average total functional disability (ADL and IADL) score was $1.5 \pm 3.7$ (range, $0-17$ ). The average log-transformed functional disability score was $0.33 \pm 0.75$ (range, 0-2.83). The percentages of dependent responses for the ADL/IADL items are shown in Table 1. Functional status was generally better for younger ( $<75$ years) and educated (vs. non-educated) respondents, while gender differences in functional status varied by item. Women reported being better at household activities such as cooking and doing laundry, while men reported needing less help with other IADL items including going out, using transportation, shopping, managing money, and using the telephone. No gender differences were seen in responses to ADL items, aside from defecation (Table 1 ).

Two IADL items and all seven ADL items were relatively free of item bias according to gender, age group, and educational level (Table 2). Eight IADL items appeared to have practically meaningful item-level biases, with ORs $>2.0$. Men were 7.69 times more likely to report needing help with preparing meals (95\% confidence interval [CI], 5.14-11.50) and 2.55 times more likely to report needing help doing laundry (95\% CI, 1.86-3.49) than women matched for overall functional disability. In contrast, men were less likely to report needing help going outside to a nearby location $(\mathrm{OR}=0.43$; 95\% CI, 0.28-0.67), using transportation $(\mathrm{OR}=0.21$; 95\% CI, 0.14-0.31), shopping ( $\mathrm{OR}=0.40$; 95\% CI, 0.25-0.63), managing money $(\mathrm{OR}=0.36$; $95 \% \mathrm{CI}, 0.26-0.51)$, and using the telephone ( $\mathrm{OR}=0.48 ; 95 \% \mathrm{CI}, 0.34-0.68)$. Adults aged 75 years or older were more likely to report dependence in managing money $(\mathrm{OR}=2.04 ; 95 \% \mathrm{CI}, 1.51-2.74)$ and using the telephone $(\mathrm{OR}=3.37$; 95\% CI, 2.36-4.81) than younger elderly people matched for overall functional status.

Seven IADL items presented meaningful item-level bias according to educational level. The item bias was similar to the results by gender in terms of preparing meals, doing laundry, using transportation, shopping, managing money, and using the telephone. However, going outside nearby was not biased according to educational level. In addition, the odds of educated people reporting that they needed help doing household chores was 2.14 times greater than that of older adults with no education (95\% CI, 1.31-3.48) (Table 2).

Men matched for functional ability reported being more disabled than women in performing household chores, preparing meals, and doing laundry, three items that comprised the household activities dimension of the IADL. Thus, this item-level bias carried over as a positive factor-level bias. As seen in Table 3, the OR of men reporting higher levels of disability in the household activities dimension subscale was 4.27 (95\% CI, 3.25-5.60) times that in women matched for overall functional disability. Regarding the cognitive activities dimension, the OR for men was 0.37 (95\% 
Table 1. Dependent rates (percentages) for each ADL and IADL task by sex, age, and educational level among older Korean adults, KLoSA baseline

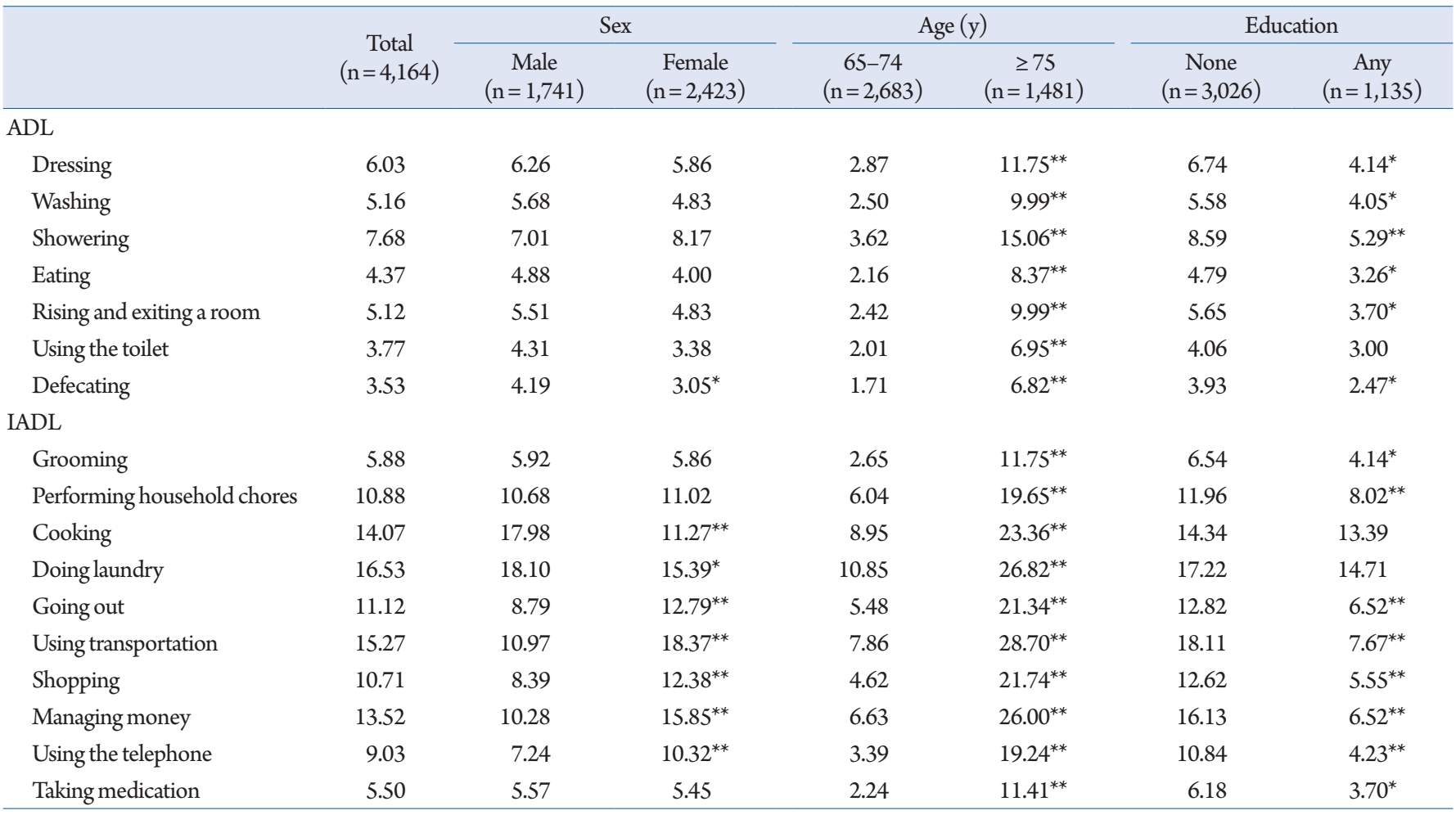

$\mathrm{ADL}$, activities of daily living; IADL, instrumental activities of daily living; KLoSA, Korean Longitudinal Study of Aging. ${ }^{*} \mathrm{p}<0.05,{ }^{* *} \mathrm{p}<0.001$ by chi-square test according to sex, age, and educational level separately.

CI, 0.29-0.46) times that of women matched for overall functional disability. The cognitive and household activities dimensions were positive factor-level biases for both age group and educational level (Table 2).

The internal consistency reliability of the full version of $\mathrm{ADL} /$ IADL was 0.96, as measured using Cronbach's alpha. The reduced 9-item $\mathrm{ADL} / \mathrm{IADL}$ that excluded the 8 IADL items with item-level bias retained an internal consistency reliability of 0.95 . The 9-item version of the $\mathrm{ADL} / \mathrm{IADL}$ showed a correlation of 0.68 with the full 17 -item version. The disability rate from the reduced 9-item $\mathrm{ADL}$ and $\mathrm{IADL}$ was $10.6 \%$, which was lower than that for the full version $(25.3 \%)$. Gender differences in disability rates were apparent in the reduced version in that women were significantly more disabled (11.4\%) than men (9.4\%). No gender differences in disability rates were detected using the full version. Older and less educated individuals were more likely to have some disability; this tendency was the same regardless of full or short version of the ADL/IADL (Table 3).

\section{DISCUSSION}

In our study, eight out of ten IADL items functioned differently among subgroups defined by gender, age, and educational level. Four of these items related to household activities, three were cognitive activities, and one item was related to physical activity. The items comprising household activities were biased in the direction of higher endorsement by men (i.e., men were more likely to rate themselves as dependent in these areas), while items comprising cognitive activities were biased toward higher endorsement among women after matching for overall functional disability. Measurement with the DIF-adjusted versions of $\mathrm{ADL}$ and $\mathrm{IADL}$ revealed statistically robust gender-based differences in disability rates even though the absolute gap in disability rate by gender was similar between original and DIF-adjusted versions of $A D L$ and IADL.

In previous studies examining gender-based item response bias, DIF was detected in items related to bathing, ${ }^{23)}$ shopping, ${ }^{11,15)}$ performing household chores, ${ }^{11)}$ using the telephone, ${ }^{15)}$ caring for others, ${ }^{11)}$ doing laundry, ${ }^{19)}$ and taking medication. ${ }^{19)}$ However recent studies applying DIF analysis to $\mathrm{ADL}$ and $\mathrm{IADL}$ were exclusively performed in Western settings, where the gendered division of household labor is not as skewed as in the Asian context. In this study, we found item-level bias in all of those items as well as in items related to managing money and using transportation, which are cognitive activities. In addition, we found item-level bias in the 
Table 2. DIF by gender, educational level, and age

\begin{tabular}{lccc}
\hline & Men & Age $\geq 75 y$ & Education \\
\hline ADL & & & \\
Dressing & 1.15 & 0.98 & 0.87 \\
Washing hands and face & 1.20 & 0.56 & 1.86 \\
Bathing & 0.64 & 0.92 & 1.30 \\
Feeding self & 1.40 & 0.67 & 1.08 \\
Walking across room & 1.44 & 0.74 & 1.24 \\
Using toilet & 1.15 & 0.54 & 1.38 \\
Defecating \& urinating & 1.83 & 0.56 & 0.54 \\
IADL & & & \\
Grooming & 0.99 & 0.90 & 1.16 \\
Doing light housework & 1.55 & 0.58 & $\mathbf{2 . 2 0}$ \\
Preparing meals & $\mathbf{7 . 8 3}$ & 0.51 & $\mathbf{4 . 9 0}$ \\
Laundry & $\mathbf{3 . 0 5}$ & $\mathbf{0 . 4 4}$ & $\mathbf{4 . 2 5}$ \\
Going outside nearby & $\mathbf{0 . 4 3}$ & 0.93 & 0.66 \\
Using transportation & $\mathbf{0 . 2 0}$ & 1.57 & $\mathbf{0 . 2 1}$ \\
Shopping & $\mathbf{0 . 4 0}$ & 1.97 & $\mathbf{0 . 3 7}$ \\
Managing finances & $\mathbf{0 . 3 7}$ & 1.74 & $\mathbf{0 . 3 0}$ \\
Using the telephone & $\mathbf{0 . 4 9}$ & $\mathbf{3 . 0 3}$ & $\mathbf{0 . 2 9}$ \\
Taking medication & 1.03 & 1.87 & 0.83 \\
ADL dimension* & & & \\
Self-care dimension & 1.27 & 0.61 & 1.22 \\
Mobility dimension & 1.26 & 0.83 & 1.03 \\
IADL dimension $\dagger$ & & & \\
Physical activities dimension & 0.66 & 1.13 & 0.70 \\
Cognitive activities dimension & $\mathbf{0 . 3 5}$ & $\mathbf{2 . 8 0}$ & $\mathbf{0 . 2 2}$ \\
Household activities dimension & $\mathbf{4 . 0 1}$ & 0.68 & $\mathbf{2 . 5 1}$ \\
\hline
\end{tabular}

Bold text represent a significant difference $(\mathrm{OR}>2.0$ or $\mathrm{OR}<0.5)$.

$\mathrm{DIF}$, differential item functioning; $\mathrm{ADL}$, activities of daily living; IADL, instrumental activities of daily living.

*Self-care include dressing, washing, showering, eating, using toilet, defecating and urinating; and Mobility include getting up and exiting a room.

${ }^{\dagger}$ Physical activities include grooming, going out nearby; Cognitive activities, using transportation, managing money, using the telephone, taking medication; and Household activities, performing household chores, preparing meals, doing laundry, shopping.

direction of endorsement of further disability in women for the physical activity of getting around outside. Comparison of our results with those of previous studies (Supplementary Table S1) showed that more IADL items in the Korean older adults showed gender-specific response biases than those in other populations. To our knowledge, this is the first such report among East Asian countries. The differential item responses to household activities were more pronounced in this study than the gendered DIF reported in Singapore. ${ }^{19)}$

We also observed item response bias according to age group ( $<75$ vs. $\geq 75$ years) for doing laundry and using the telephone. The older age group was less likely to endorse disability in doing
Table 3. Comparisons of disability rates from the full $\mathrm{ADL} / \mathrm{IADL}$ and DIF-adjusted reduced scales

\begin{tabular}{|c|c|c|c|}
\hline & \multirow[b]{2}{*}{$\mathrm{n}$} & \multicolumn{2}{|c|}{ Disability rate } \\
\hline & & Full scale & $\begin{array}{l}\text { DIF-adjusted } \\
\text { short scale }\end{array}$ \\
\hline Total & 4,164 & 25.3 & 10.6 \\
\hline \multicolumn{4}{|l|}{ Sex } \\
\hline Male & 1,741 & 24.4 & $9.4^{*}$ \\
\hline Female & 2,423 & 26.0 & 11.4 \\
\hline \multicolumn{4}{|l|}{ Age (y) } \\
\hline $65-74$ & 2,683 & $16.7^{* *}$ & $5.2^{* *}$ \\
\hline $75-84$ & 1250 & 35.4 & 16.0 \\
\hline$\geq 85$ & 231 & 71.0 & 43.3 \\
\hline \multicolumn{4}{|l|}{ Educational level } \\
\hline Elementary or below & 3,026 & $27.8^{* *}$ & $12.1^{* *}$ \\
\hline Middle school & 407 & 19.9 & 6.6 \\
\hline High school or over & 728 & 18.0 & 6.3 \\
\hline \multicolumn{4}{|l|}{ Men } \\
\hline \multicolumn{4}{|l|}{ Age (y) } \\
\hline $65-74$ & 1,211 & $18.9^{* *}$ & $5.6^{* *}$ \\
\hline $75-84$ & 463 & 32.4 & 15.1 \\
\hline$\geq 85$ & 67 & 67.2 & 37.3 \\
\hline \multicolumn{4}{|l|}{ Educational level } \\
\hline Elementary or below & 921 & $27.3^{* *}$ & $11.1^{*}$ \\
\hline Middle school & 249 & 23.3 & 8.0 \\
\hline High school or over & 570 & 20.2 & 7.2 \\
\hline \multicolumn{4}{|l|}{ Women } \\
\hline \multicolumn{4}{|l|}{ Age (y) } \\
\hline $65-74$ & 1,472 & $14.8^{* *}$ & $4.9^{* *}$ \\
\hline $75-84$ & 787 & 37.2 & 16.5 \\
\hline$\geq 85$ & 164 & 72.6 & 45.7 \\
\hline \multicolumn{4}{|l|}{ Educational level } \\
\hline Elementary school or below & 2,105 & $28.0^{* *}$ & $12.6^{* *}$ \\
\hline Middle school & 158 & 14.6 & 4.4 \\
\hline High school or over & 158 & 10.1 & 3.2 \\
\hline
\end{tabular}

Full scale include 17 items of ADL and IADL; and DIF-adjusted short scale include 9 items of ADL and IADL.

ADL, activities of daily living; IADL, instrumental activities of daily living; DIF, differential item functioning.

${ }^{*} \mathrm{p}<0.05,{ }^{* *} \mathrm{p}<0.01$ by chi-square test.

laundry and more likely to do so in using the telephone. This finding is similar to the gender item response differences for these items. This similarity might be due to the high correlation between age and gender: $64.2 \%$ of the adults aged 75 years or older were women, indicating that women were more likely to be older than men. In previous studies, the items that differed in item response according to age included preparing meals ${ }^{19)}$ and performing household chores. ${ }^{177}$ Fleishman et al. ${ }^{15)}$ compared DIF according to combined gender and age groups, observing item-level bias in 
shopping, doing light housework, and managing money in young men and shopping and using the telephone in middle-aged men compared to those in older me. The results of these studies demonstrated the tendency for items showing DIF of age and gender to overlap.

Previous studies have not identified IRT according to educational level. However, as many of the IADL items include cognitive activities and education level is low among the current older generations in countries such as Korea that have experienced recent rapid growth, it is important to investigate IRT according to educational attainment. In Korea, the current older generations, who reached adulthood during the period of rapid modernization after prolonged war during their school-age years, have relatively little exposure to westernized formal educational systems. ${ }^{24)}$ Older women are particularly likely to have lower levels of formal education due to unequal opportunities in the educational system, as reflected by the fact that $69.5 \%$ of older women the present study had not completed an elementary school education. The similarities between DIF according to educational level and according to gender reflect the strong correlation between gender and education level.

The current full versions of $\mathrm{ADL}$ and $\mathrm{ADDL}$ do not accurately capture disability rates because of their acknowledged biases in item responses by gender, age, and education level. In addition, the current scales do not clearly show differences between groups. The higher disability rates in women's cognitive activities are due to the latent gender construct reflected in these items; if this is not considered, measurements of disability in women would be exaggerated. Likewise, men report greater difficulties with household activities items because of the latent construct reflected in these items and, if not taken into consideration, the disability rates of men are also exaggerated. Aside from the 8 items with high DIF, the reliability of these scales was high and the gender differences in disability rates based on $\mathrm{ADL} / \mathrm{IADL}$ responses to the other 9 items were more accurate than those reported by the previous full version. Women have higher rates of disability than men and are more likely to have daily disabilities than are men of the same age $\mathrm{e}^{25)}$ due to musculoskeletal disease morbidity and pain sensitivity. In this study, women were more likely to report limitations, use of assistance, and a greater degree of disability, particularly among IADL categories. However, these gender differences were largely explained by differences in disability-related health conditions. The comorbid conditions associated with disability in this cohort, which were predominantly musculoskeletal, neurodegenerative, and psychological in origin, were generally more prevalent among women than in men. ${ }^{25)}$ One limitation of our study was the time gap since the KLoSA baseline survey was performed (2006), 13 years ago. Many older citizens now own and use smartphones since their invention in 2007. Thus, technology compliance should be also considered when interpreting our study results.

As culture and the degree of social infrastructure development differ across countries, responses to ADL and IADL are highly context-specific. Unless corrected for, cross-national variations in disability rates may, therefore, reflect item-response bias rather than real differences in disability levels. Older adults in Korea have experienced strong gender segregation from a young age and have grown up in a social structural environment with limited educational opportunities, particularly for older women. These cultural aspects are reflected in IRT-based research of older Korean populations. Further IRT research on the ability to perform everyday activities in societies with diverse cultural backgrounds is needed to allow more accurate measurement of disabilities across cultures. In particular, comparisons of disability rates by country should separately assess household and cognitive activities from the IADL scale, or should completely exclude these two dimensions when comparing $\mathrm{ADL}$ and IADL item measurements. If possible, a culture-neutral $\mathrm{ADL}$ and $\mathrm{IADL}$ measurement that does not require DIF analyses should be developed.

\section{CONFLICT OF INTEREST DISCLOSURES}

The researchers claim no conflicts of interest.

\section{SUPPLEMENTARY MATERIALS}

Supplementary materials can be found via https://doi.org/10. 4235/agmr.19.0047.

\section{REFERENCES}

1. Lawton MP. The functional assessment of elderly people. J Am Geriatr Soc 1971;19:465-81.

2. Lawton MP. Aging and performance of home tasks. Hum Factors 1990;32:527-36.

3. LaPlante MP. The demographics of disability. Milbank Q 1991;69 Suppl 1-2:55-77.

4. Allen SM, Mor V, Raveis V, Houts P. Measurement of need for assistance with daily activities: quantifying the influence of gender roles. J Gerontol 1993;48:S204-11.

5. Park S, Park BS. Testing reliability and measurement invariance of K-ADL. Health Soc Welf Rev 2017;37:98-124.

6. Won CW, Rho YG, SunWoo D, Lee YS. The validity and reliability of Korean Instrumental Activities of Daily Living (K-IADL) scale. J Korean Geriatr Soc 2002;6:273-80.

7. Won CW. Korea activities of daily living scale and Korea instru- 
mental activities of daily living scale. J Korean Geriatr Soc 2002;6:1-10.

8. Won CW, Yang KY, Rho YG, Kim SY, Lee EJ, Yoon JL, et al. (2002). The development of Korean activities of daily living (K-ADL) and Korean instrumental activities of daily living (K-IADL) scale.J Korean Geriatr Soc 2002;6:107-20.

9. Kim CS. Gender differentials of economic resources in old age. Korea J Popul Stud 2003;26:59-77.

10. Gadermann AM, Guhn M, Zumbo BD. Estimating ordinal reliability for Likert-type and ordinal item response data: a conceptual, empirical, and practical guide. Pract Assess Res Eval 2012;17:1-13.

11. Cabrero-Garcia J, Lopez-Pina JA. Aggregated measures of functional disability in a nationally representative sample of disabled people: analysis of dimensionality according to gender and severity of disability. Qual Life Res 2008;17:425-36.

12. LaPlante MP. The classic measure of disability in activities of daily living is biased by age but an expanded IADL/ADL measure is not.J Gerontol B Psychol Sci Soc Sci 2010;65:720-32.

13. Saliba D, Orlando M, Wenger NS, Hays RD, Rubenstein LZ. Identifying a short functional disability screen for older persons. J Gerontol A Biol Sci Med Sci 2000;55:M750-6.

14. Chan KS, Kasper JD, Brandt J, Pezzin LE. Measurement equivalence in $\mathrm{ADL}$ and IADL difficulty across international surveys of aging: findings from the HRS, SHARE, and ELSA.J Gerontol B Psychol Sci Soc Sci 2012;67:121-32.

15. Fleishman JA, Spector WD, Altman BM. Impact of differential item functioning on age and gender differences in functional disability.J Gerontol B Psychol Sci Soc Sci 2002;57:S275-84.

16. Teresi JA, Cross PS, Golden RR. Some applications of latent trait analysis to the measurement of ADL. J Gerontol 1989;44:S196204.
17. Ng TP, Niti M, Chiam PC, Kua EH. Physical and cognitive domains of the Instrumental Activities of Daily Living: validation in a multiethnic population of Asian older adults. J Gerontol A Biol Sci Med Sci 2006;61:726-35.

18. Spector WD, Fleishman JA. Combining activities of daily living with instrumental activities of daily living to measure functional disability.J Gerontol B Psychol Sci Soc Sci 1998;53:S46-57.

19. Niti M, Ng TP, Chiam PC, Kua EH. Item response bias was present in instrumental activity of daily living scale in Asian older adults. J Clin Epidemiol 2007;60:366-74.

20. Cole SR, Kawachi I, Maller SJ, Berkman LF. Test of item-response bias in the CES-D scale: experience from the New Haven EPESE study.J Clin Epidemiol 2000;53:285-9.

21. Holland PW, Wainer H. Differential item functioning. London: Routledge; 2012.

22. Crane PK, Gibbons LE, Jolley L, van Belle G. Differential item functioning analysis with ordinal logistic regression techniques. DIFdetect and difwithpar. Med Care 2006;44(11 Suppl 3):S115-23.

23. Teresi JA, Ocepek-Welikson K, Kleinman M, Cook KF, Crane PK, Gibbons LE, et al. Evaluating measurement equivalence using the item response theory log-likelihood ratio (IRTLR) method to assess differential item functioning (DIF): applications (with illustrations) to measures of physical functioning ability and general distress. Qual Life Res 2007;16 Suppl 1:4368.

24. Yi G. Cultural capital and social capital of the Korean elderly. J Korea Gerontol Soc 2005;25:1-21.

25. Murtagh KN, Hubert HB. Gender differences in physical disability among an elderly cohort. Am J Public Health 2004;94:140611. 